\title{
Wild Bumblebee (Bombus) Diversity and Nosema (Microsporidia: Nosematidae) Infection Levels Associated with Lowbush Blueberry (Vaccinium angustifolium) Production and Commercial Bumblebee Pollinators
}

\author{
Sara L. Bushmann, Francis A. Drummond, Lee A. Beers, and Eleanor Groden \\ School of Biology and Ecology, University of Maine, 5722 Deering Hall, Orono, ME 04469, USA \\ Correspondence should be addressed to Sara L. Bushmann, sara_bushmann@umit.maine.edu
}

Received 4 April 2012; Revised 24 August 2012; Accepted 27 August 2012

Academic Editor: Zachary Huang

Copyright (C) 2012 Sara L. Bushmann et al. This is an open access article distributed under the Creative Commons Attribution License, which permits unrestricted use, distribution, and reproduction in any medium, provided the original work is properly cited.

\begin{abstract}
The primary objective was to determine if the prevalence of Nosema bombi infection is higher for wild bumblebees (Bombus spp.) caught in lowbush blueberry growing areas with a history of commercial bumblebee use than for bumblebees caught in areas without a history of commercial bumblebee use. Additionally, we wished to determine relative Bombus species abundances and diversity in blueberry growing regions. Over two years we caught, identified to species, and dissected 767 bumblebees. Light microscopy revealed overall infection levels of 5.48\%. The history of commercial bumblebee use had no relation to infection levels. Bumblebee species diversity and field location had significant relationships to infection $\left(r^{2}\right.$ adjusted $=0.265$; species diversity $F_{(1,22)}=6.848, P=0.016$; field region $\left.F_{(1,22)}=5.245, P=0.032\right)$. The absence or presence of one species, Bombus terricola, appears to determine the relationship between species diversity and infection. The data show $B$. terricola decline in sampled regions and almost half of the collected B. terricola were infected with Nosema. The commercial species, B. impatiens, shows an increase in abundance, but with a $6.9 \%$ proportion infection. Molecular confirmation of the infecting species was ambiguous, suggesting a need for future clarification of the infecting species.
\end{abstract}

\section{Introduction}

Native bumblebees (Bombus spp.) serve as valuable pollinators for the lowbush blueberry (Vaccinium angustifolium Aiton) fields in Eastern Maine and Maritime Canada and Quebec. Typically queens are the predominant foragers during blueberry bloom in Maine [1]. They are known to be effective pollinators due to their ability to forage in cool or rainy conditions [2], which commonly occur during lowbush blueberry bloom in Maine [3], and their use of sonication to remove pollen grains from the Vaccinium poricidal anthers [4]. As pollinators of lowbush blueberry, Bombus spp. surpass Apis mellifera (L.), the most commonly used commercial pollinator, in terms of purity of pollen load (plant fidelity), flower visitation rates, flower handling time, pollen deposition, and percentage of foragers collecting pollen as opposed to nectar [1,5-7].

Since the early 1990s, commercially reared bumblebees have been available for blueberry growers in Maine [8] and this option appears to be a boon to farmers wishing to enhance pollination by complementing or replacing A. mellifera. Furthermore, the species provided in commercial colonies, Bombus impatiens Cresson, is technically native to Maine, although it is not known whether the source of commercial genotypes is native to Maine. Many farmers wish to use a native species and many express a hope to populate their fields with subsequent generations of wild bumblebees [9].

The use of commercial bumblebees in Maine lowbush blueberry has not reached the levels of honeybees, peaking 
in the mid-2000s, and is since responsible for imported pollination services in about 1.3 to 2.0 percent of the blueberry acreage in Maine [1]. This roughly translates into 400-600 acres of lowbush blueberry fields pollinated with 2000-2400 colonies of commercial bumblebees. These colonies are placed in groups of four, referred to as a quad, directly in the fields where they usually remain until the end of the colony life cycle. Gynes (female reproductives) and males are often produced from the commercial colonies [9].

Consequences of the placement of commercially reared bees in areas with contact to wild bees have been documented $[10,11]$. In a greenhouse situation, Colla et al. [12] showed that pathogens were more prevalent in wild bumblebees located near tomato greenhouses using commercial bumblebees than in more distant wild bumblebee populations. In this study, we examine possible consequences of using commercial bumblebees as pollinators of lowbush blueberry. Specifically, we ask: do commercial B. impatiens used in the Maine lowbush blueberry agroecosystem, though technically a native bee, impact disease incidence and relative abundances of naturally occurring Bombus species?

We chose to answer this question, in part, by looking at the prevalence of the microsporidian genus Nosema in wild bumblebees in and around Maine blueberry fields. We expected to find Nosema bombi (Fantham and Porter [13]), an obligate intracellular parasite that commonly occurs in North American bumblebees [14]. Nosema bombi was one of the pathogens hypothesized by Colla et al. [12] to have jumped from commercial bumblebees to wild bumblebees foraging near greenhouses. It has been suggested that a European strain of N. bombi transferred from commercially reared bumblebees has been responsible for the decline of three species of bumblebees [15] including B. terricola Kirby, a bumblebee historically found in moderate-to-high population densities in blueberry fields in Maine [16]. Cameron et al. [17] reported B. terricola to currently have reduced abundance in relation to historical records while also showing an increased level of $N$. bombi infection (albeit based on a small sample size) in comparison to species without population declines.

The effects of N. bombi infection on colony and individual health have proven difficult to assess. Studying laboratory reared B. terrestris (L.) inoculated with N. bombi, Steen [18] reported low levels of colony success due to poor brood survival of inoculated queens. Likewise, Otti and SchmidHempel [19] found infected males had reduced sperm levels and infected queens had decreased ability to mate. In terms of colony success, infected colonies appeared to have reduced population size [20]. In contrast, Whittington and Winston [21] found no significant effects of N. bombi infection on colony size (B. occidentalis Greene) or amount of brood, although the authors suggest the experimental time (10 weeks) and/or the colony growth limitations due to greenhouse conditions may have obscured effects seen in older or free-ranging colonies. In general, the evidence seems to point to detrimental colony and individual health, (see also $[14,22]$ ) with the understanding that host species [23] and colony genetics [22] might influence the severity of effects due to infection.

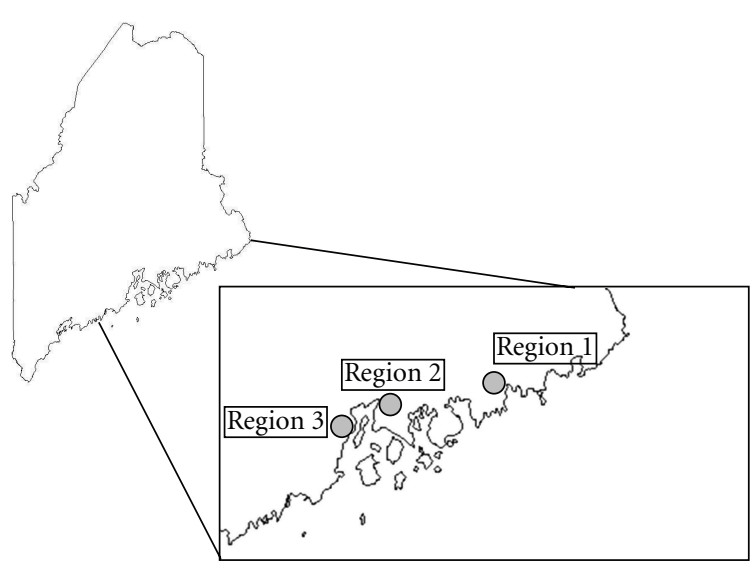

Figure 1: Map of the three lowbush blueberry regions in Maine in which the field sites are located: Region 1: 8 fields, Region 2: 8 fields +1 unpaired field, and Region 3: 8 fields.

The primary objective of this study was to determine if the prevalence of N. bombi infection is higher in bumblebees caught in lowbush blueberry growing areas with a history of commercial bumblebee use than in bumblebees sampled in areas without a history of commercial bumblebee use. Based on the work of Colla et al. [12], we hypothesized that a history of commercial bumblebee use would result in higher levels of $N$. bombi infection. If the history of commercial bumblebee use could not explain the pattern of Nosema infection, our second objective was to determine if measurable bee or field characteristics were predictive of infection occurrences. Our final objective was to determine relative Bombus species abundances and the species diversity in blueberry growing regions. We hypothesized that due to the use of commercial bumblebees for pollination services, the relative abundance of $B$. impatiens in Maine blueberry growing regions has increased.

\section{Materials and Methods}

Twenty-four lowbush blueberry fields located in Washington, Hancock, Waldo and Knox counties of Eastern Maine were identified according to their history of commercial pollinator use by personal historical observation or grower interview (Drummond pers. comm.). A field was considered to have a commercial bumblebee history if at any point in time, commercial bumblebees were used in that field for at least one growing season since 1995. A field was considered to not have a commercial bumblebee history if commercial bumblebees had never been placed in that field. Some of the fields without a history of bumblebee use were routinely stocked with honeybees while others were not stocked with commercial bees of any kind, relying instead on wild bee pollinators.

Fields were paired according to the bumblebee history: a yes being paired with a no. The fields were located in three major blueberry regions of coastal Maine (Figure 1), with 
each pair located in the same general region. The distance between pairs of fields ranged from $1.5 \mathrm{~km}$ to $17.8 \mathrm{~km}$, with a mean distance of $8.9 \mathrm{~km}$. Six pairs of fields were visited for wild bumblebee collecting in 2009 and another independent six pairs of fields were sampled in 2010. All three regions were visited each year. One nonpaired field located in the town of Amherst ME (Hancock Co) that had been previously stocked with commercial bumblebees was visited in 2009.

The fields ranged in size from 2.2 to 20.5 hectares, although five of the fields were contiguous with other blueberry fields that were not included in the study. For these fields, the study collecting area was measured as opposed to total field size, which can run over 500 hectares of continuous blueberry landscape. The remaining collecting sites were isolated, discrete blueberry fields bordered mainly by forest. The collecting area in these fields was the entire forest delineated field.

2.1. Field Management. Production practices varied from field to field. Farmers and field managers were contacted to determine how each individual field had been managed during one complete growing cycle that ended with the year of collection. The fields varied according to the types and extent of pesticide applications and pruning methods (Table 1). Lowbush blueberry pruning methods typically create a two-year cycle with one year of vegetative growth followed by a flowering and then fruiting year. Not all fields were bearing fruit during the collection year.

2.2. Bee Collections. Collection primarily took place in July and August when most foraging bees are workers. This minimizes the capture of queens. At the start of each collection period, a route was planned along field edges where noncrop plants were in flower. In Maine, lowbush blueberry bloom is from mid-May to mid-June. The planned route was covered twice per visit at a steady, slow pace. All observed foraging bumblebees were individually caught with a sweep net and then transferred to a clean $15 \mathrm{~mL}$ plastic centrifuge tube. The tubes were stored on ice until the bees could be placed in a freezer $\left(-20^{\circ} \mathrm{C}\right)$ where they were held until dissection. In order to best document the diversity of bees foraging in the fields, each field was visited 2-3 times over 4-6 weeks, with a goal of capturing 30 bees per field. In a subset of the fields $(n=13)$, the common name of the flower each bee was caught on was recorded. Some of the common names included several species. For example, "goldenrod" was recorded without differentiating among the possible species. But the recorded names did distinguish the plants at the generic level.

A third year of collecting was conducted in 2011 to develop a relationship between bee abundance, measured as the number of bees collected per unit time and Bombus species richness. One hundred twenty-five bees were collected from thirteen new blueberry fields that did not have active commercial colonies of B. impatiens or a history of use of such colonies. This collection was conducted in a similar fashion to the two previous years, but the collection bouts were timed. All collecting was done by one person who walked at a regular pace along a predetermined path along

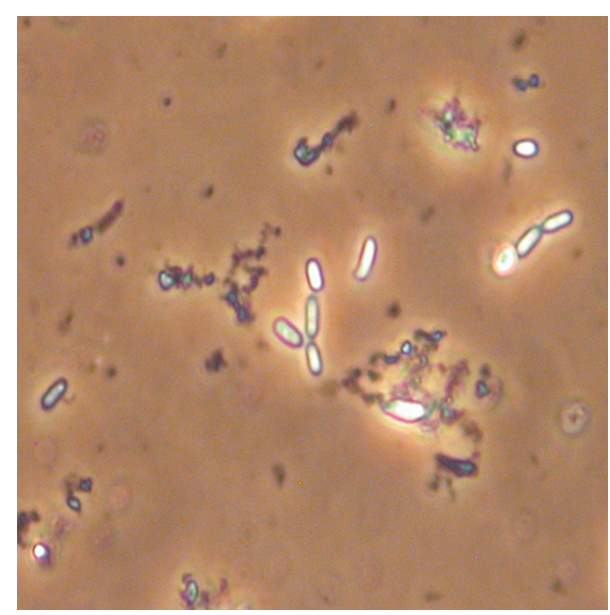

FIgURE 2: Image of spores classified as Nosema by phase-contrast light microscopy. The smaller elongate spores measure $4.5 \times 2.0$ microns. PCR with the Nbombi-SSU-Jfl/Jrl primer pair did not produce a detectable amplicon for this bee.

flowering vegetation. The collecting took place from 5 August to 19 August. Collected bees were identified to species, but not inspected for Nosema infection.

2.3. Species Identification, Bee Age, Size, and Sex. All bees were sexed and identified to species using published keys $[24,25]$ and the online keys available through http://www .discoverlife.org/. For a subset (248 individuals caught in 2009), the right front wing was collected. Using electronic calipers, the length of the marginal cell was measured to $0.01 \mathrm{~mm}$ in order to estimate bee size [26]. The degree of wing wear was used to estimate age using a method adapted from Cartar [27].

2.4. Bee Dissection. Each bee collected in 2009 and 2010 was dissected and the gut tissues examined under phase-contrast microscopy at 400x magnification in order to determine if the bee was infected with Nosema. A bee was scored positive for infection if two or more microsporidian spores were seen (as in Figure 2). The criteria for spore identification were based on size, shape, and reflectivity as described by MacFarlane et al. [14] and Larsson [23].

The bees were dissected by two different methods. Most of the 2009 bees were opened along the dorsal side of the abdomen. Small (about $2 \mathrm{~mm}$ ) lengths of the mid and hindgut were removed, placed on a slide in a drop of distilled water and crushed with a coverslip. The remainder of the bee was then refrozen $\left(-20^{\circ} \mathrm{C}\right)$. In 2010 , the entire abdomen was removed and ground with a pestle in a $1.7 \mathrm{~mL}$ microcentrifuge tube with $200 \mu \mathrm{L}$ of distilled water. A sample of the resulting solution was examined under the phase contrast microscope. This second method of bee dissection was undertaken in order to detect spores that may not have been present in the gut tract but were present in other abdominal tissues [23]. The second method of dissection also prepared the bee for molecular identification of the microsporidian. 
TABle 1: Production practices associated with the 25 sampled lowbush blueberry fields, 2009-2010. The field was given a score for each production practice based on the treatment the field received for one complete growing cycle ( 2 calendar years) ending with the bumblebee collection year. These practices were included in the linear model to examine field characteristics and Nosema prevalence.

\begin{tabular}{lcc}
\hline $\begin{array}{l}\text { Production } \\
\text { practice }\end{array}$ & Treatment & $\begin{array}{c}\text { Number of } \\
\text { fields }\end{array}$ \\
\hline \multirow{3}{*}{ Pruning } & Mow & 3 \\
& Burn & 8 \\
& Mow and burn & 6 \\
& No chemical control & 8 \\
\hline \multirow{3}{*}{ Pest control } & Standard pesticides & 8 \\
& Reduced risk pesticides & 4 \\
Herbicides & Standard and reduced risk pesticides & 13 \\
\hline \multirow{2}{*}{ Fungicides } & Yes & 17 \\
& No & 8 \\
\hline \multirow{2}{*}{ Insecticides } & Yes & 15 \\
& No & 10 \\
\hline
\end{tabular}

2.5. Molecular Confirmation of Infection. All 2010 bee samples that scored positive for Nosema with microscopic observations were centrifuged for 5 minutes $(16,100 \mathrm{~g})$, the supernatant discarded and the homogenate frozen at $-80^{\circ} \mathrm{C}$. All of the 2009 Nosema-positive and some of the 2009 Nosema-negative refrozen dissected bees were thawed and their abdomens removed and similarly ground, centrifuged, and subject to DNA extraction. DNA was extracted using a DNeasy Plant Mini Kit (Qiagen) and amplified using the genus-specific primer SSUrRNA-fl/rlc and species-specific primer Nbombi-SSU-Jfl/Jrl (Table 2) following the protocols of Klee et al. [28] using an Eppendorf thermocycler. The former primer pair contains sequences conserved in $N$. bombi, N. apis, and $N$. ceranae, while the later primer is specific to $N$. bombi small subunit rRNA sequences [28]. PCR products were visualized with electrophoresis on a $1.4 \%$ agarose gel stained with ethidium bromide. Samples were considered positive for Nosema if a band was visible at the expected fragment length (Table 2). Fragment size was confirmed with a $100 \mathrm{bp}$ ladder (Promega). A subsample ( $n=8$ ) of $222 \mathrm{bp}$ fragments from the PCR products of the genus specific primer SSUrRNA-fl/rlc were extracted from $1.4 \%$ agarose gels stained with GelStar (Lonza), purified with a QIAquick Gel Extraction Kit (Quiagen) and sequenced on an Applied Biosystems (ABI) model 377 Sequencer at the University of Maine DNA Sequencing Facility. Edited and aligned sequences were compared with those deposited in GenBank.
2.6. Analysis. For all analyses of relationships between bee host or field factors and infection prevalence, infection prevalence was based on the proportion of bees determined to be Nosema positive with microscopic examination. As an indication of possible pathogen spread from a point-source such as one of the fields with a history of bumblebee use, we conducted a Mantel test [29] that compared matrices of differences in field infection prevalence and differences in geographic distances between fields. We used a randomization test with 1,000 iterations [30]. The distances were measured in a straight line from the center of each field using Google Earth (6.1.0.5001). When the most direct route between fields crossed a body of water greater than $1.5 \mathrm{~km}$ (a flight distance based on B. terrestris L.; [31]) the shortest land route was measured. The closest fields were $1.2 \mathrm{~km}$ apart and the most distant were $154.2 \mathrm{~km}$ apart. Only the 24 paired fields were used for this analysis. Our question for this test was do fields that are closer together have similar infection levels?

Bumblebee species diversity for each field was calculated as Shannon's index [32]. Because of the difficulty of identifying male bees of the subgenus Psithyrus (12 individuals), all bees of this subgenus, including the three females, were grouped and considered one species for diversity calculations. Species richness was defined as the total number of species found in each field. With the 2011 bee species abundance data linear regression [33] was used to develop a predictor of density from species richness so that the effect of estimated Bombus spp. density on Nosema infection could be assessed $\left(r^{2}=0.740 ; P=0.0003\right)$.

A plant generic diversity (Shannon's index, [32]) and generic richness measure (total number of genera represented by the bee catch) were obtained for the subset of 13 fields with known flower types bees were caught on. These measures represent the diversity and richness of the beevisited floral resources observed in each field and are not an exhaustive list of flowering vegetation, but represent the most common and preferred floral resources. We used linear regression analysis [33] to evaluate the relationship between plant generic richness and diversity measures and Nosema infection levels for the thirteen fields.

Considering the full data set of 25 fields, we used stepwise linear regression [33] to select models to examine relationships between 12 field characteristics and field-level (averaged across individual bees) Nosema infection levels. The field characteristics included bee species richness, bee species diversity, history of commercial bumblebee use, location (region 1, 2, or 3, see Figure 1), rotational stage (fruit bearing year or not), area, distance to the nearest field with commercial bumblebee use, and the six production practices listed in Table 1 . We used a mixed procedure with the probability to leave and enter at $\alpha=0.250$ and with square root transformed infection proportions. In this model, Bombus species richness was used as a proxy for Bombus spp. density as described above.

All general statistics and regression analyses were performed with JMP version 8.0.2 [34]. Mantel tests were conducted using PC-ORD, version 6 [30]. 
TABLE 2: Primers used for PCR amplification of ribosomal RNA.

\begin{tabular}{|c|c|c|c|c|}
\hline Name & Strand direction & Sequence $\left(5^{\prime}-3^{\prime}\right)$ & Annealing temp. (C) & Expected fragment size \\
\hline \multirow{2}{*}{ SSUrRNA-fl } & \multirow{2}{*}{ Forward } & CACCAGGTTG & \multirow{4}{*}{48} & \multirow{4}{*}{222} \\
\hline & & ATTCTGCCT & & \\
\hline \multirow{2}{*}{ SSUrRNA-rlc } & \multirow{2}{*}{ Reverse } & GTTACCCGTC & & \\
\hline & & ACTGCCTTG & & \\
\hline \multirow{2}{*}{ Nbombi-SSU-Jfl } & \multirow{2}{*}{ Forward } & CCATGCATGTT & \multirow{4}{*}{50} & \multirow{4}{*}{323} \\
\hline & & TTTGAAGATTATTAT & & \\
\hline \multirow{2}{*}{ Nbombi-SSU-Jrl } & \multirow{2}{*}{ Reverse } & CATATATTTTTA & & \\
\hline & & AAATATGAAACAATAA & & \\
\hline
\end{tabular}

Based on Klee et al., 2006 [28], developed from the complete rRNA N. bombi consensus sequence, Accession no. AY741110.

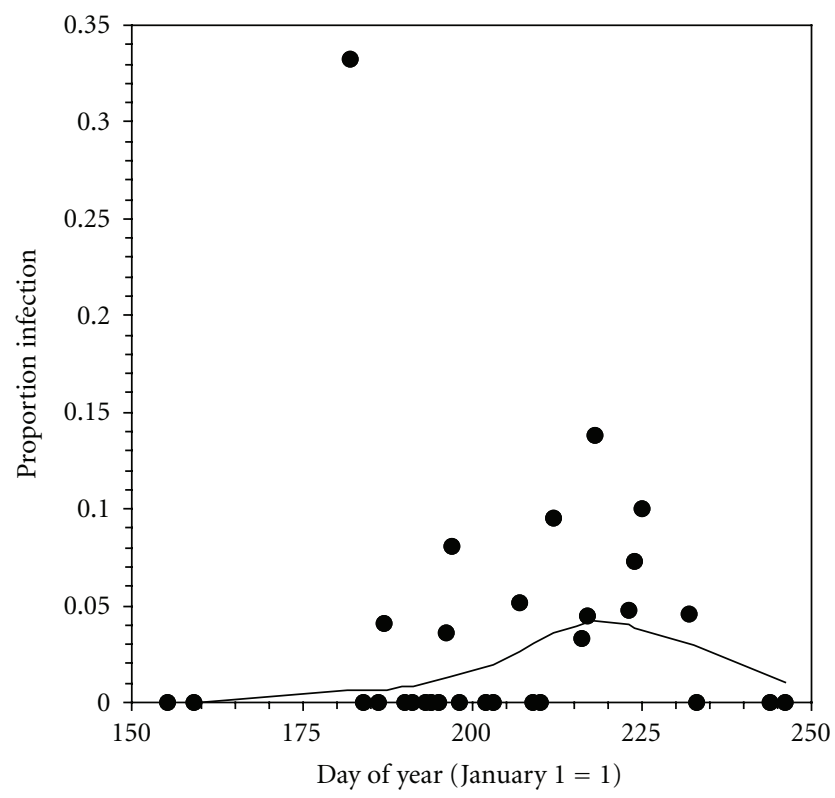

Figure 3: Proportion of bumblebees sampled from Maine blueberry fields showing Nosema infection as determined by light microscopy. Proportions shown by day of the year between the sampling period, 4 June-3 September, 2009-2010 (data from two years pooled). The high of $33.33 \%$ is from a day with a total catch of three bees, one of which scored positive for Nosema spp.

\section{Results}

Over two years, 767 bumblebees were caught, identified to species, and dissected. Of these, 42 bees were positive for Nosema infection according to light microscopy inspection, resulting in an overall $5.48 \%$ infection level (Table 3 ). Neither sex (646 females, 37 female Nosema positive), nor dissection method (297 bees by method one, 13 Nosema positive), nor year of capture (373 bees caught in 2009 with 16 infected) explained the patterns of infection (Fisher's Exact, $P=0.66, P=0.33, P=0.21$, resp.). Each bee was given an ordinal rank for the day of the year on which it was caught (Julian day). While not significant at the $\alpha=0.05$ level $\left(\chi^{2}=3.48, d f=1, P=0.06\right)$ Nosema infections showed a trend toward higher incidence as the foraging season progressed (Figure 3), and then declined at the end of the summer. Sampling did not continue in the Autumn.
TABLE 3: Nosema infection in bumblebee species collected in Maine blueberry fields over two years (2009-2010) based on microscopic and molecular examination.

\begin{tabular}{lccc}
\hline Species & $\begin{array}{c}\text { Number } \\
\text { positive*/ } \\
\text { number caught }\end{array}$ & \% Infection* & $\begin{array}{c}\text { Fraction of } \\
\text { positive* bees } \\
\text { without } \\
\text { amplification } \\
\text { (see text) }\end{array}$ \\
\hline B. terricola & $6 / 13$ & 46.2 & 0.33 \\
B. perplexus & $3 / 18$ & 16.67 & 0 \\
B. impatiens & $7 / 102$ & 6.86 & 0.72 \\
Psithyrus & $1 / 15$ & 6.67 & 0 \\
B. vagans & $10 / 175$ & 5.71 & 0.5 \\
B. ternarius & $14 / 374$ & 3.74 & 0.57 \\
B. bimaculatus & $1 / 68$ & 1.47 & 1 \\
* Based on visual assessment under 400x magnification with phase contrast \\
microscopy.
\end{tabular}

3.1. Field History. Of the 25 fields visited for bee collections, 13 were originally identified as having a history of commercial bumblebee use and 12 were identified as no history of commercial bumblebee use. When interviewing growers about management practices, however, one no commercial bumblebee field was changed to a commercial bumblebee field as that grower indicated that a manager of an adjoining field had used commercial bumblebees in the past. This resulted in 14 commercial bumblebee fields (462 bees caught) and 11 no commercial bumblebee fields (295 bees caught). Of these remaining eleven no commercial bumblebee fields, none were located with an adjoining field under different management. Twenty-five Nosema positive bees came from commercial bumblebee fields and 17 came from no commercial bumblebee fields. There was no difference in the proportion of infected bees according to the history of using commercial bumblebees (Fisher's Exact Test, $P=0.87$ ). This conclusion does not change when considering the original categorization of the fields related to commercial bumblebee use.

Six fields had active commercial bumblebee colonies during the collection periods. There was no indication that those fields had levels of infection that differed significantly from the other nineteen fields (Student's two-tailed test, $t=$ $-0.12, d f=23, P=0.45$ ) or from the 10 fields with no 


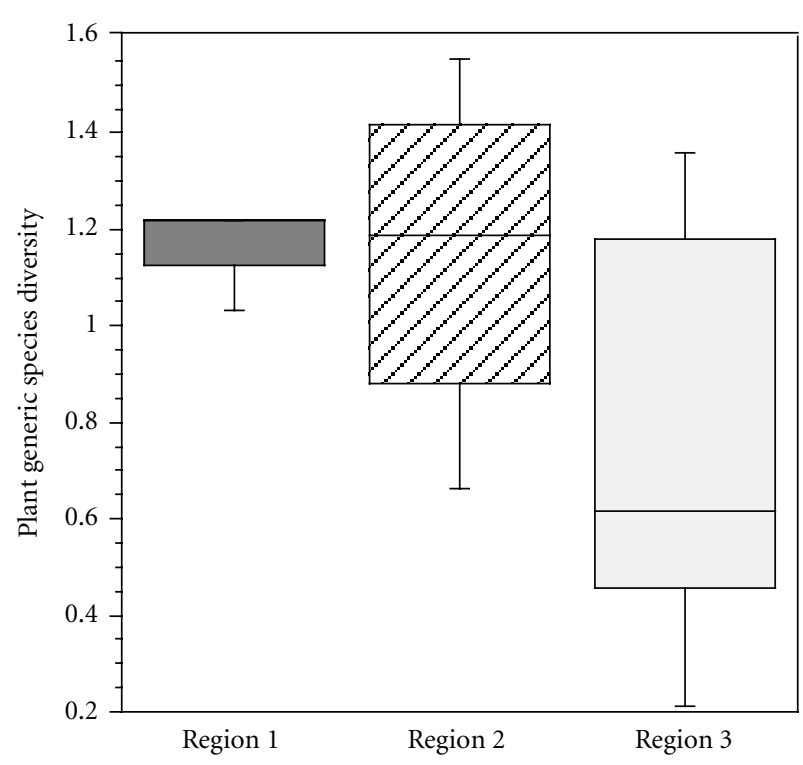

Figure 4: Median, range, and upper and lower quartiles of generic species diversity of plants from which bumblebees were collected along the edge of blueberry fields in three regions in Maine. Based on a subset of 13 fields. Region 1, $n=3$; Region 2, $n=4$; Region 3, $n=6$.

history of commercial bumblebees (Student's two-tailed test, $t=0.15, d f=16, P=0.51)$.

3.2. Bee Age, Size, and Species. For a subsample of 248 bees, age and size measurements were obtained. Logistic regression reveals no relation between these two parameters and infection (size: Wald's $\chi^{2}=.55, d f=1, P=0.46$; age: Wald's $\chi^{2}=2.57, d f=3, P=0.46$; size $*$ age: Wald's $\chi^{2}=2.92$, $d f=3, P=0.40)$. When considering all 767 bees, it was apparent that infection was not evenly distributed across species (Table 3 ). The proportion of infected B. terricola was significantly higher than the other bee species (Fisher's Exact Test, $P=0.0002$ ). We did find one infected individual of the subgenus Psithyrus, in contrast to Larsson [23].

3.3. Field Characteristics. For the subset of thirteen fields for which we calculated plant generic diversity and generic richness, we found no significant linear trend relating floral generic richness and field-level infection level $(P=0.23)$. However, plant generic diversity showed a significant, negative relationship with infection level $\left(F_{(1,11)}=4.70, P=\right.$ 0.05 ) and differed according to collection region (Figure 4$).$

Out of the 12 field characteristics considered as possible predictors for the occurrence of Nosema in the 2009 and 2010 sampling, only Bombus species diversity and blueberry growing region were significantly associated with infection. Together, these two characteristics account a little more than $26 \%$ of the variation in infection $\left(r^{2}\right.$ adjusted $=0.27$; species diversity $F_{(1,22)}=6.85, P=0.016$; field region $\left.F_{(1,22)}=5.25, P=0.03\right)$. The proportions of infected bees for collecting regions 1,2 , and 3 were $0.04 \pm 0.02,0.04 \pm$ 0.01 , and $0.08 \pm 0.02$ (mean $\pm \mathrm{SE})$, respectively.

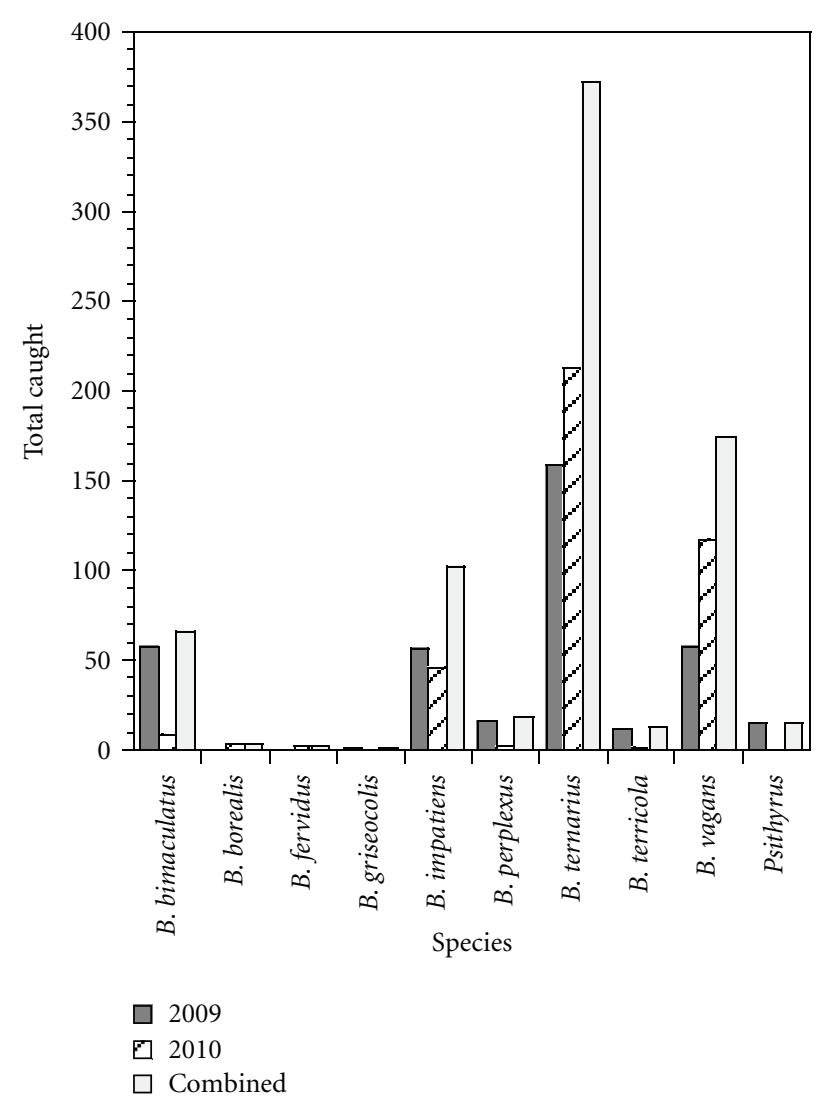

Figure 5: Bumblebees caught in and around lowbush blueberry fields over two years, by year and combined ( $n=767$ bees).

3.4. Bee Species Diversity. Ten species were identified in the total collection, including 15 individuals of the subgenus Psithyrus found in five different fields (Figure 5). Figure 5 includes those $B$. impatiens found in fields with active commercial colonies. Although all bees were caught at flowers and commercial bees were not targeted, some of $B$. impatiens (13.3\% of total catch) were likely commercial bees. When all $B$. impatiens caught in fields with active commercial colonies were removed from the data set, only 35 individuals remained, which represents $5 \%$ of the resulting total. Bombus ternarius (Say) was by far the most abundant bee over the two years, making up $48.5 \%$ of the total collection and $42.6 \%$ and $54.1 \%$ of the 2009 and 2010 collections, respectively (Figure 5). The species diversity calculated for the total catch of each year declined over the two years of collecting by nearly 30\% (Shannon's Index for $2009=1.61 ; 2010=1.14$ ). In order to identify factors that influence species diversity, we compared the relationship between infection and species diversity when $B$. terricola was excluded from the data to the full data set. When all thirteen B. terricola caught over two years are removed, the relationship changes from a significant one, to insignificant (Figure 6).

In order to examine long-term trends in relative abundances, we retrieved measures from lowbush blueberry fields from 1961-63 [16] and 1997 and 1998 (Drummond, unpubl. data, $n=34$ lowbush blueberry fields). The 1960s were well before the widespread use of commercial bumblebees, which 


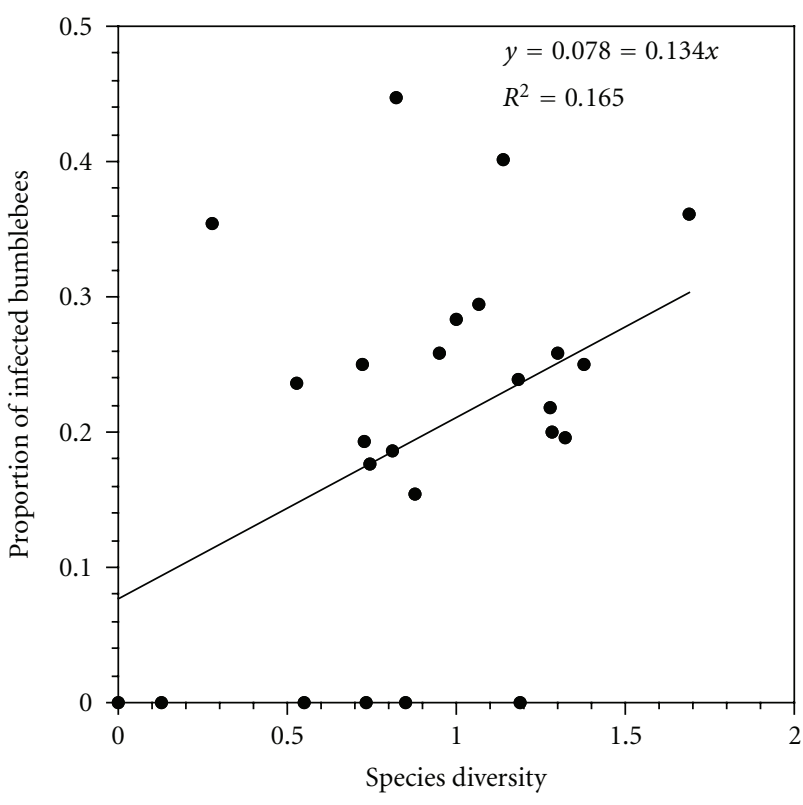

(a) Model: $d f=1, F=4.56, P=0.04$

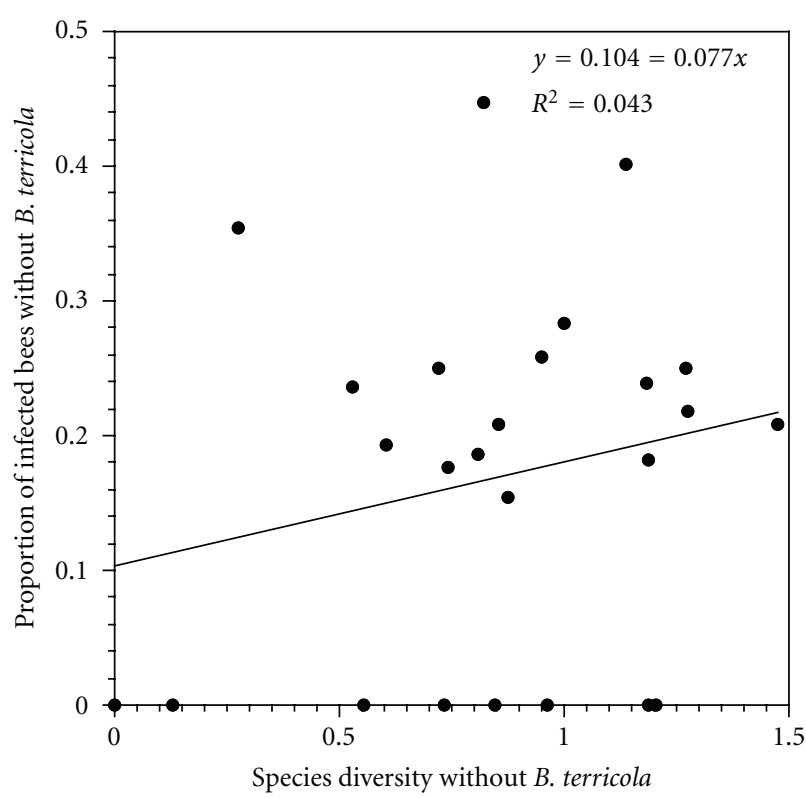

(b) Model: $d f=1, F=1.02, P=0.32$

Figure 6: Nosema infection (square root proportion infected) of bumblebees relative to species diversity in 25 blueberry fields in 2009 and 2010 with and without B. terricola.

were not adopted by Maine blueberry growers until the mid-1990s. We also collected 377 bumblebees in 2011 from blueberry fields within Region 2 of the disease collections. The relative abundances are shown in Figure 7.

3.5. Evidence for Pathogen Introduction. The results of the Mantel randomization test give no indication that fields with similar infection levels are geographical neighbors $(P=$ 0.16). A second Mantel test conducted using the logarithm of both variables (proportion infected and distance) gives

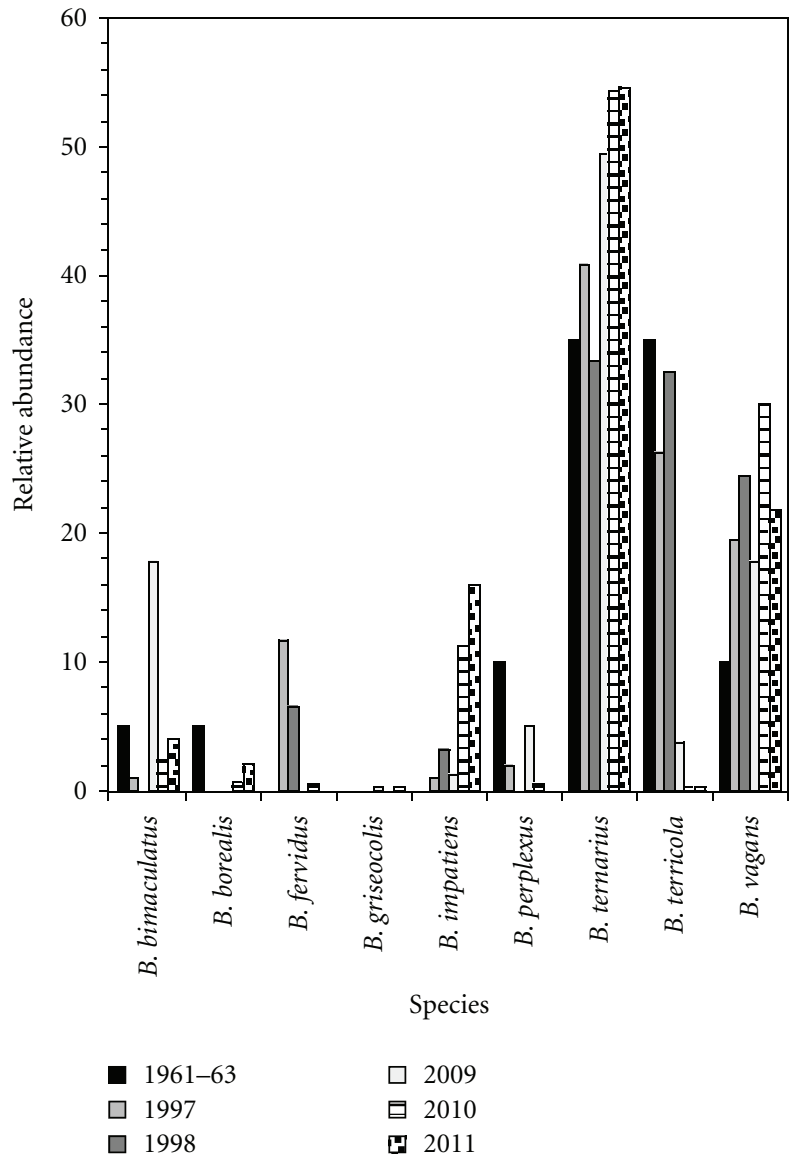

Figure 7: Relative abundances of bumblebee species found in blueberry fields for five years: 1961-63, 1997, 1998, 2009, 2010, and 2011. Psithyrus not shown. B. impatiens from fields with active commercial bumblebee colonies not included. Data for 1961-63 from Boulanger et al. [16].

no indication of a nonlinear relationship between infection levels and geographic similarity $(P=0.16)$.

The molecular confirmation of the infecting species was conducted on 41 of the 42 bees scored as Nosema-positive by light microscopy. One bee was omitted from this analysis due to damage during storage. Of these 41 bees, only 21 could be confirmed as being infected with Nosema bombi according to our protocol. For these 21 bees, PCR results showed amplification of DNA at the expected fragment lengths for both the general primers designed for detection of Nosema spp. (SSUrRNA-fl/rlc) and the primers specific for Nosema bombi (Nbombi-SSU-Jfl/Jrl). The remaining 20 bees had no amplification with the species-specific primers. For these bees, however, the results with the more general primers were ambiguous. Amplification products of expected size were present, but often accompanied with fragments of different lengths not associated with the primer. Furthermore, six of the bees scored as negative by light microscopy gave the same result: no amplification with the Nbombi-SSUJfl/Jrl primer pair, yet positive, with multiple-bands evident, for the SSUrRNA-fl/rlc primer pair. Of the eight samples sequenced, five sequences were consistent with $N$. bombi, 
one consistent with Nosema spp., and two gave unreadable results. Of the five consistent samples, four were from the bees confirmed as $N$. bombi and one from those with evident multiple bands with the SSUrRNA-fl/rlc primer pair. The one sample consistent with Nosema spp. and the two samples with unreadable results were from those with multiple bands with the general primers. Because of the difficulty of isolating bands at the target fragment length when multiple bands were present (resulting in unreadable results) no other samples were prepared for sequencing.

\section{Discussion}

The data provide no support for our prediction that the use of commercial bumblebees (B. impatiens) in Maine's blueberry fields has increased the prevalence of Nosema infection in those fields that have a history of commercial bumblebee use. Although our sample size is low, the power of our test is high, 0.879 , for detecting large differences in infection (difference of a 0.5 proportion in Nosema spp. prevalence with a minimal detectable odds ratio of 3 and a significance level of 0.05). Therefore, we can conclude that there was no evidence of large differences in prevalence of Nosema spp. in wild bumblebees due to the use of commercial bumblebees by farmers. The total infection level of $5.48 \%$ does not indicate that coastal areas of blueberry production in Maine have an elevated prevalence of infection. This prevalence is lower than that found in a recent survey of bumblebees in Massachusetts [35], but within the ranges found in multistate surveys $[17,36]$.

However, when looking at individual species it is apparent that not all species are equally likely to harbor similar levels of infection. Our results show that B. terricola has a higher rate of infection than the other Bombus species, although our results are based on a low sample size for this species (13 individuals, Table 3 ). If we assume that infection is independent among collected individuals, then the likelihood of a sample of $13 \mathrm{~B}$. terricola (proportion infected $46.2 \%$ ) coming from a bumblebee community with an average prevalence rate of $5.48 \%$ is very low $(P=0.0000313$, based upon the cumulative binomial distribution). This is a pattern also observed in a recent North American-wide survey [17] and also in a related recent analysis of geographic distributions of Nosema [37]. In western North America, $B$. terricola along with two species of the same subgenus, $B$. occidentalis and B. affinis Cresson, appear to be declining both in their abundance and range [15, 17, 38, 39]. This decline has been hypothesized to be a result of pathogen spillover from commercial bumblebees [12, 15, 40, 41]. Thorp [15] suggests that in the early 1990s commercial North American bees were reared in Europe and subsequently infected with a virulent biotype of $N$. bombi that was transmitted to wild bees in the US and Canada when colonies from these populations were used for pollination. To the best knowledge of one of us (F. A. Drummond), commercial bees first started being used in Maine lowbush blueberry fields around 1995, a time that would roughly coincide with Thorp's timeline for the introduction of European strains of N. bombi. With this study, however, we find no evidence of pathogen spillover when looking at the geographic data set comprising fields from all three regions. Across the blueberry growing regions, we find no clustering of infection. Based on pathogen identification with light microscopy, we find the only suggestion of pathogen spillover is the clustering of the pathogen within one species, which we consider to be insufficient evidence of commercial bumblebee contribution to Nosema prevalence in wild bumblebee populations due to the fact that this short-term study is unable to document longer-term disease/host population dynamics.

We found two field characteristics that help explain the distribution of Nosema infection across the three blueberry regions. Bumblebee species diversity showed the strongest influence and region of field location as a lesser predictor. We suggest that the species diversity is primarily driven by the presence or absence of $B$. terricola (Figure 6) and that this effect is a result of the nearly $50 \%$ infection prevalence of that species.

The fields in the most southern region (Region 3) of our sampling area have a higher mean prevalence of infection than the fields from the other regions. All regions have at least one field with no infected bees, but Region 3 also contains fields with the highest infection prevalence that occurred in the study. The causes of this cluster are not clear. According to our regression analysis that examined twelve field characteristics, only species diversity also explained prevalence of Nosema infection. Comparison of species diversity means by region reveals no differences so this measure does not account for the cluster of infections in Region 3. However, the data from the subset of 13 fields did show a negative relationship between plant generic diversity and field infection prevalence. The fields from Region 3 in this subset do show the lowest levels of plant generic diversity found in this study (Figure 4). This could be a spurious relationship, but two factors lower diversity; a small number of species and/or the dominance of a few species in the population. Both of these conditions would force bumblebees to forage on the same flower types, which, if Nosema is transmitted on the flower itself, as with other pathogens [42], easier disease transmission between colonies could occur. Further research will be needed to confirm and clarify this relationship.

Our analyses rely on light microscopy to determine the presence of Nosema infection in Bombus. This approach is limited to identifying pathogens to the genus level only. Spores that were of the correct size and shape for N. bombi overlap with those of other Nosema species ( $N$. ceranae, $N$. apis) $[43,44]$. To identify the infecting agent at the species level, we attempted to isolate the pathogenic DNA for molecular analysis. Of the 41 bees showing positive Nosema infection via light microscopy and subjected to molecular analysis, only 21 had amplification with the species-specific primer pair Nbombi-SSU-Jfl/Jrl. The other 20 lacked clear amplification, but were clearly infected with Nosema-like spores under the light microscope. This may reflect a variant of $N$. bombi which does not amplify with this speciesspecific primer, or suggest the presence of a different Nosema species. The more general primers, SSUrRNA-fl/rlc, amplify conserved regions of rRNA commonly held across N. bombi, 
N. apis, N. ceranae [28], and other Nosema and Vairimorpha species. Amplification with PCR from these primers was evident in all 41 bees. Six bees that scored Nosema-negative with light microscopy also showed amplification with these primers (but with no amplification with the NbombiSSU-Jfl/Jrl primers), a situation which may indicate sample contamination or amplification of nontarget DNA. We cannot conclude, with any confidence, the species of infecting Nosema from these PCR results.

The cross-infectivity of $N$. apis and bumblebees has been questioned [23] and while there is evidence that $N$. ceranae has crossed from honeybees to bumblebees in South America [45], such a host jump has not been documented elsewhere. $N$. ceranae appears to have recently crossed from the Asian honeybee (Apis cerana F.) to the European honeybee (Apis mellifera L.) $[44,46]$ and is now found globally in infected honeybees [47]. Every year blueberry growing regions of Maine are stocked with rented honeybees, which could prove a source of either N. ceranae or N. apis. However, given the low likelihood of bumblebees successfully infected with either N. apis or N. ceranae, and given the known, widespread occurrence of $N$. bombi [17] and lack of evidence of other microsporidian infective agents in bumblebees, we consider it reasonable to treat all observed infective agents as Nosema and most likely $N$. bombi. Further research identifying optimal primers in order to reliably sequence isolated gene fragments of the infective agent is warranted.

Exactly half of the 20 bees that did not produce amplification with the Nbombi-SSU-Jfl/Jrl primers came from fields with a history of commercial bumblebee use and half came from fields without the bumblebee use. All collection regions and five species were represented by this group of 20 bees that showed no amplification with the species specific primers. Only two of the originally determined six Nosema-positive B. terricola are represented by this group (Table 3 ).

Our final objective was to examine relative species abundances in Maine's blueberry growing region after about 17 years of importation of $B$. impatiens as pollinators. Our recent surveys compared with historic relative abundances (Figure 7) suggest that since the use of commercial bumblebees, the relative abundance of $B$. impatiens has increased. While some Bombus species have remained relatively stable, B. terricola and to a lesser extent B. fervidus (F.), have pronounced reduced abundances. B. ternarius, while always abundant, also shows an upward trend. The shift in abundance of $B$. impatiens suggests that queens reared by commercial colonies may be successfully overwintering and founding their own colonies. Bombus impatiens was not reported in Maine or Maritime Canada in the 1960s [16], (Drummond unpubl. data). Mark-recapture studies of new queens conducted by Stubbs and Drummond [6] have shown that commercial $B$. impatiens queens will overwinter successfully in Maine. This is not an unreasonable frequent occurrence, as commercial colonies often stay in lowbush blueberry fields through the colony lifecycle. While Figure 7 suggests species shifts within bumblebee communities are occurring, this data does not support or refute the concern that bumblebees as a whole are in decline in Maine lowbush blueberry growing regions as described for other regions globally [48]. However, shifts in biodiversity of bumblebees could have impacts on the ecosystem services provided by these important wild pollinators $[49,50]$. This should be a top priority for future research.

\section{Acknowledgments}

The authors would like to thank Ms. Tamara Levitsky, Ms. Jennifer Lund, and Ms. Judith Collins for their generous assistance with multiple aspects of this study. Mr. David Simmons and Dr. Joyce Longcore gave valuable contributions with the lab work. They also thank Drs. Andrei Alyokhin, Dave Yarborough, and Alison Dibble from the University of Maine for their helpful discussions, and Ms. Kalyn Bickerman and two anonymous reviewers for reviewing the paper and making suggestions for its improvement. They acknowledge numerous student workers notably led by Keren Zucker, Anna Delong, Cody Martel, and Brittany Cortel for their help with bee collections. They thank Dr. Lee Solter for assistance with pathogen identification and dissection technique. This project is funded by the University of Maine, School of Biology and Ecology, and an USDA NIFA fellowship awarded to S. Bushmann.

\section{References}

[1] F. A. Drummond, "Commercial bumble bee pollination of lowbush blueberry," International Journal of Fruit Science, vol. 12, pp. 216-231, 2012.

[2] B. Heinrich, Bumblebee Economics, Harvard University Press, Cambridge, Mass, USA, 2nd edition, 2004.

[3] F. A. Drummond, "Honeybees and blueberry pollination," University of Maine Cooperative ExtensionWild Blueberry Bulletin, No. 29, 2002.

[4] S. L. Buchmann, "Buzz pollination in angiosperms," in Handbook of Experimental Pollination Biology, C. E. Jones and R. J. Little, Eds., pp. 73-113, Van Nostrand Reinhold, New York, NY, USA, 1983.

[5] T. L. Whidden, "The fidelity of commercially reared colonies of Bombus impatiens cresson (Hymenoptera: Apidae) to lowbush blueberry in Southern New Brunswick," Canadian Entomologist, vol. 128, no. 5, pp. 957-958, 1996.

[6] C. S. Stubbs and F. A. Drummond, "Bombus impatiens (Hymenoptera: Apidae): an alternative to Apis mellifera (Hymenoptera: Apiclae) for lowbush blueberry pollination," Journal of Economic Entomology, vol. 94, no. 3, pp. 609-616, 2001.

[7] S. K. Javorek, K. E. Mackenzie, and S. P. Vander Kloet, "Comparative pollination effectiveness among bees (Hymenoptera: Apoidea) on lowbush blueberry (Ericaceae: Vaccinium angustifolium)," Annals of the Entomological Society of America, vol. 95, no. 3, pp. 345-351, 2002.

[8] H. H. W. Velthuis and A. van Doorn, "A century of advances in bumblebee domestication and the economic and environmental aspects of its commercialization for pollination," Apidologie, vol. 37, no. 4, pp. 421-451, 2006.

[9] C. S. Stubbs, F. A. Drummond, and D. Yarborough, "Commercial bumble bee, Bombus impatiens, management for lowbush blueberry," Wild blueberry fact sheet No. 302 (Bulletin No. 2421), University of Maine Cooperative Extension Publication, pp.1-4, 2001. 
[10] S. Niwa, H. Iwano, S. I. Asada, M. Matsuura, and K. Goka, "A microsporidian pathogen isolated from a colony of the European bumblebee, Bombus terrestris, and infectivity on Japanese bumblebee," Japanese Journal of Applied Entomology and Zoology, vol. 48, no. 1, pp. 60-64, 2004.

[11] J. C. Stout and C. L. Morales, "Ecological impacts of invasive alien species on bees," Apidologie, vol. 40, no. 3, pp. 388-409, 2009.

[12] S. R. Colla, M. C. Otterstatter, R. J. Gegear, and J. D. Thomson, "Plight of the bumble bee: pathogen spillover from commercial to wild populations," Biological Conservation, vol. 129, no. 4, pp. 461-467, 2006.

[13] H. B. Fantham and A. Porter, "The morphology, biology and economic importance of Nosema bombis, n. sp., parasitic in various humble bees (Bombus spp.)," Annals of Tropical Medicine and Parasitology, vol. 8, pp. 623-638, 1914.

[14] R. P. MacFarlane, J. J. Lipa, and H. J. Liu, "Bumble bee pathogens and internal enemies," Bee World, vol. 76, pp. 130-148, 1995.

[15] E. Evans, R. Thorp, S. Jepson, and S. H. Black, "Status review of three formerly common species of bumble bee in the subgenus Bombus," The Xerces Society for Invertebrate Conservation, 2012, http://www.xerces.org/yellow-banded-bumble-bee/.

[16] L. W. Boulanger, G. W. Wood, E. A. Osgood, and C. O. Dirks, "Native bees associated with the low-bush blueberry in Maine and Eastern Canada," University of Maine Agricultural Experiment Station, Technical Bulletin, vol. 26, p. 22, 1967.

[17] S. A. Cameron, J. D. Lozier, J. P. Strange et al., "Patterns of widespread decline in North American bumble bees," Proceedings of the National Academy of Sciences of the United States of America, vol. 108, no. 2, pp. 662-667, 2011.

[18] J. J. M. V. D. Steen, "Infection and transmission of Nosema bombis in Bombus terrestris colonies and its effect on hibernation, mating and colony founding," Apidologie, vol. 39, no. 2, pp. 273-282, 2008.

[19] O. Otti and P. Schmid-Hempel, "Nosema bombis: a pollinator parasite with detrimental fitness effects," Journal of Invertebrate Pathology, vol. 96, no. 2, pp. 118-124, 2007.

[20] O. Otti and P. Schmid-Hempel, "A field experiment on the effect of Nosema bombis in colonies of the bumblebee Bombus terrestris," Ecological Entomology, vol. 33, no. 5, pp. 577-582, 2008.

[21] R. Whittington and M. L. Winston, "Effects of Nosema bombi and its treatment fumagillin on bumble bee (Bombus occidentalis) colonies," Journal of Invertebrate Pathology, vol. 84, no. 1, pp. 54-58, 2003.

[22] P. Schmid-Hempel and R. Loosli, "A contribution to the knowledge of Nosema infections in bumble bees, Bombus spp," Apidologie, vol. 29, no. 6, pp. 525-535, 1998.

[23] J. I. R. Larsson, "Cytological variation and pathogenicity of the bumble bee parasite Nosema bombis (Microspora, Nosematidae)," Journal of Invertebrate Pathology, vol. 94, no. 1, pp. 1-11, 2007.

[24] T. B. Mitchell, "Bees of the eastern United States. II," Technical Bulletin, North Carolina Agricultural Experiment Station, vol. 152, pp. 1-557, 1962.

[25] T. M. Laverty and L. D. Harder, "The bumble bees of eastern Canada," Canadian Entomologist, vol. 120, no. 11, pp. 965967, 1988.

[26] R. E. Owen, "Body size variation and optimal body size of bumble bee queens (Hymenoptera: Apidae)," Canadian Entomologist, vol. 120, pp. 19-27, 1988.

[27] R. V. Cartar, "Morphological senescence and longevity: an experiment relating wing wear and life span in foraging wild bumble bees," Journal of Animal Ecology, vol. 61, no. 1, pp. 225-231, 1992.

[28] J. Klee, W. Tek Tay, and R. J. Paxton, "Specific and sensitive detection of Nosema bombis (Microsporidia: Nosematidae) in bumble bees (Bombus spp.; Hymenoptera: Apidae) by PCR of partial rRNA gene sequences," Journal of Invertebrate Pathology, vol. 91, no. 2, pp. 98-104, 2006.

[29] B. F. J. Manly, Randomization, Bootstrapping and Monte Carlo Methods in Biology, Chapman and Hall, London, UK, 1997.

[30] B. McCune and M. J. Mefford, PC-ORD. Multivariate Analysis of Ecological Data. Version 6. MjM Software, Gleneden Beach, Oregon, Ore, USA, 2011.

[31] J. L. Osborne, A. P. Martin, N. L. Carreck et al., "Bumblebee flight distances in relation to the forage landscape," Journal of Animal Ecology, vol. 77, no. 2, pp. 406-415, 2008.

[32] C. E. Shannon, "A mathematical theory of communication," TheBell System Technical Journal, vol. 27, pp. 379-423, 1948.

[33] J. H. Zar, Biostatistical Analysis, Prentice Hall, Upper Saddle River, NJ, USA, 4th edition, 1999.

[34] S. A. S. Institute Inc, "JMP Version 8.0.2 for Macintosh," SAS Institute Inc., Cary, NC, USA, 2009.

[35] S. Gillespie, "Factors affecting parasite prevalence among wild bumblebees," Ecological Entomology, vol. 35, no. 6, pp. 737$747,2010$.

[36] C. N. Kissinger, S. A. Cameron, R. W. Thorp, B. White, and L. F. Solter, "Survey of bumble bee (Bombus) pathogens and parasites in Illinois and selected areas of northern California and southern Oregon," Journal of Invertebrate Pathology, vol. 107, no. 3, pp. 220-224, 2011.

[37] N. Cordes, F. Huang, J. P. Strange et al., "Interspecific geographic distribution and variation of the pathogens Nosema bombis and Crithidia species in United States bumble bee populations," Journal of Invertebrate Pathology, vol. 109, pp. 209-216, 2012.

[38] Committee on the Status of Pollinators in North America and National Research Council, Status of pollinators in North America, The National Academies Press, Washington, DC, USA, 2007.

[39] S. Rao, W. P. Stephen, C. Kimoto, and S. J. Debano, "The status of the "red-listed" Bombas occidentalis (Hymenoptera: Apiformes) in Northeastern Oregon," Northwest Science, vol. 85, no. 1, pp. 64-67, 2011.

[40] M. C. Otterstatter and J. D. Thomson, "Does pathogen spillover from commercially reared bumble bees threaten wild pollinators?" PLoS ONE, vol. 3, no. 7, Article ID e2771, 2008.

[41] I. Meeus, M. J. F. Brown, D. C. De Graaf, and G. Smagghe, "Effects of invasive parasites on bumble bee declines," Conservation Biology, vol. 25, no. 4, pp. 662-671, 2011.

[42] S. Durrer and P. Schmid-Hempel, "Shared use of flowers leads to horizontal pathogen transmission," Proceedings of the Royal Society B, vol. 258, no. 1353, pp. 299-302, 1994.

[43] Y. P. Chen, J. D. Evans, C. Murphy et al., "Morphological, molecular, and phylogenetic characterization of Nosema ceranae, a microsporidian parasite isolated from the European honey bee, Apis mellifera," Journal of Eukaryotic Microbiology, vol. 56, no. 2, pp. 142-147, 2009.

[44] I. Fries, "Nosema ceranae in European honey bees (Apis mellifera)," Journal of Invertebrate Pathology, vol. 103, no. 1, pp. S73-S79, 2010.

[45] S. Plischuk, R. Martín-Hernández, L. Prieto et al., "South American native bumblebees (Hymenoptera: Apidae) infected by Nosema ceranae (Microsporidia), an emerging pathogen of honeybees (Apis mellifera)," Environmental Microbiology Reports, vol. 1, no. 2, pp. 131-135, 2009. 
[46] M. Higes, R. Martín, and A. Meana, "Nosema ceranae, a new microsporidian parasite in honeybees in Europe," Journal of Invertebrate Pathology, vol. 92, no. 2, pp. 93-95, 2006.

[47] J. Klee, A. M. Besana, E. Genersch et al., "Widespread dispersal of the microsporidian Nosema ceranae, an emergent pathogen of the western honey bee, Apis mellifera," Journal of Invertebrate Pathology, vol. 96, no. 1, pp. 1-10, 2007.

[48] D. Goulson, G. C. Lye, and B. Darvill, "Decline and conservation of bumble bees," Annual Review of Entomology, vol. 53, pp. 191-208, 2008.

[49] S. Naeem, L. J. Thompson, S. P. Lawler, J. H. Lawton, and R. M. Woodfin, "Declining biodiversity can alter the performance of ecosystems," Nature, vol. 368, no. 6473, pp. 734-737, 1994.

[50] M. Loreau, S. Naeem, P. Inchausti et al., "Ecology: biodiversity and ecosystem functioning: current knowledge and future challenges," Science, vol. 294, no. 5543, pp. 804-808, 2001. 

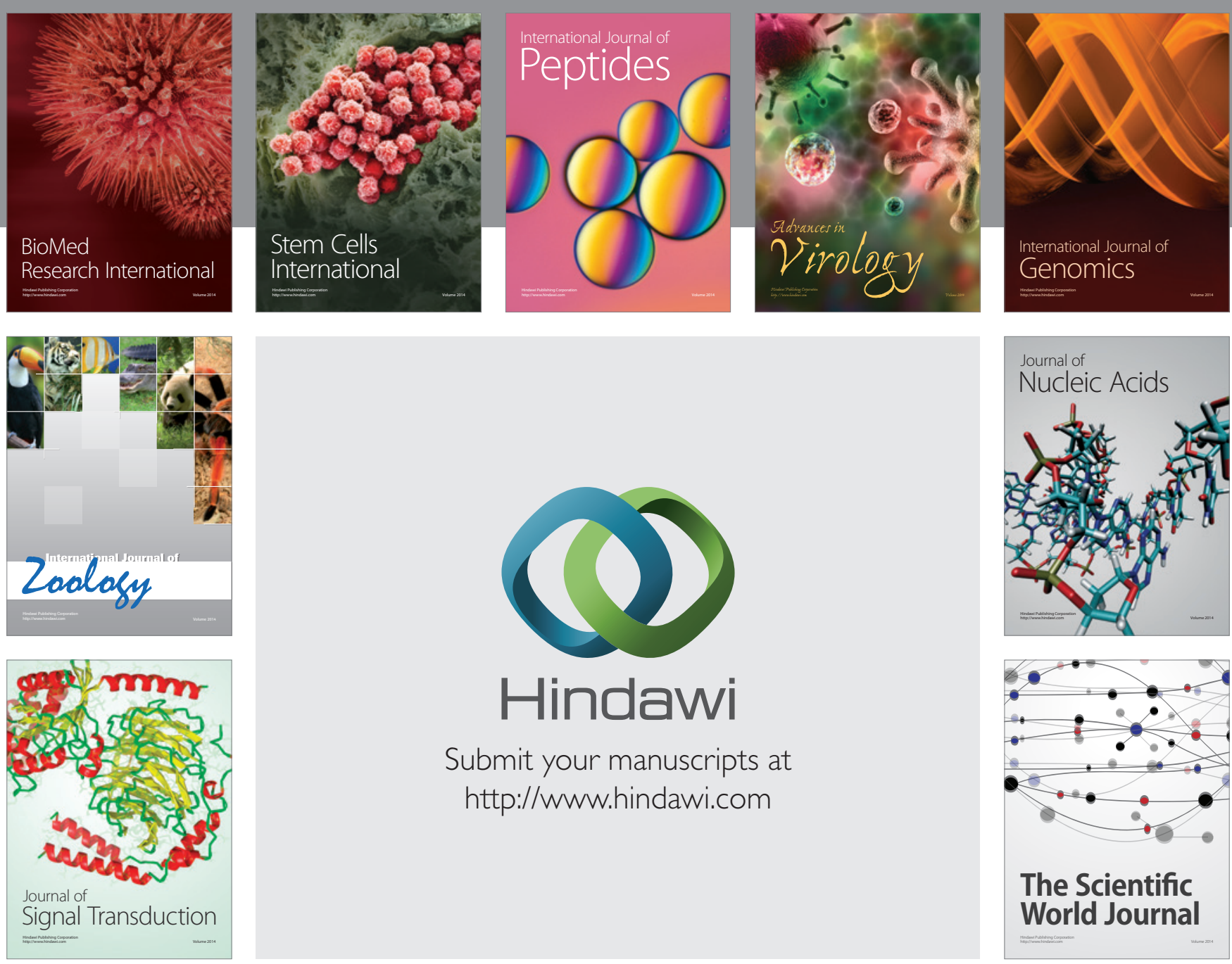

Submit your manuscripts at

http://www.hindawi.com
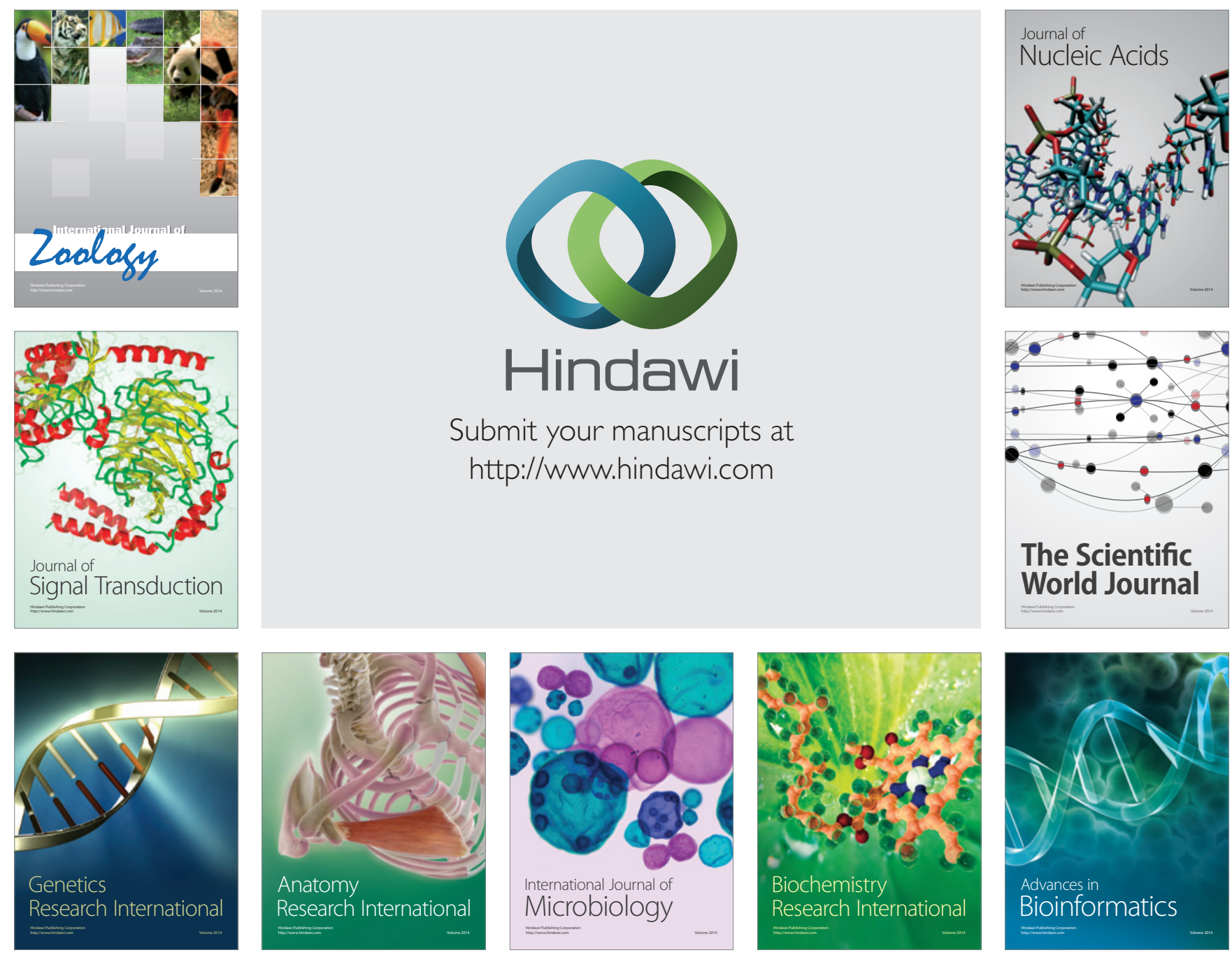

The Scientific World Journal
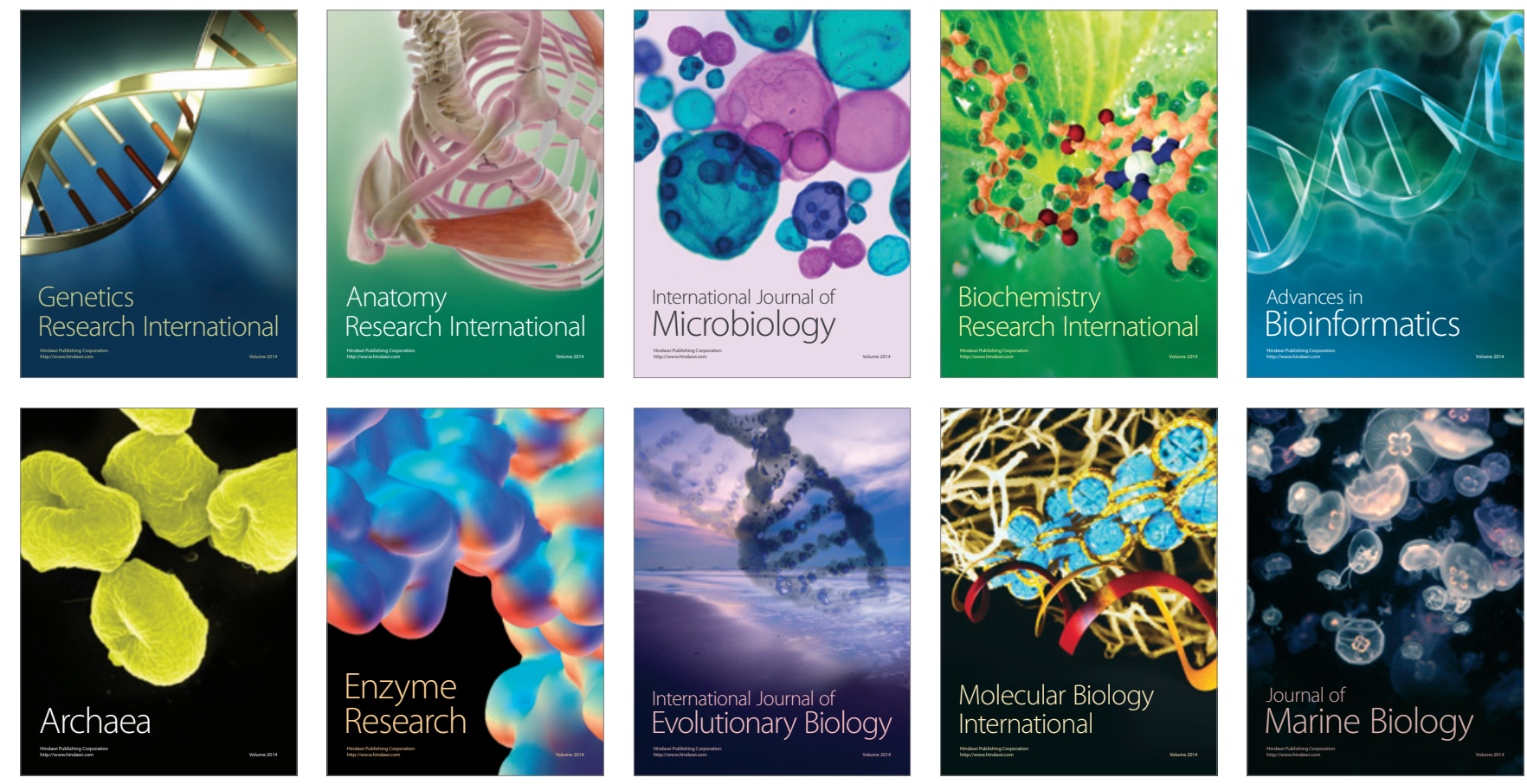